\title{
面向爬壁机器人的电极吸附阵列模型构建及优化
}

\author{
过金超 $^{1}$ 崔光照 ${ }^{2}$ 陈 旭 ${ }^{1}$ 高 瑜 ${ }^{1}$ \\ (1. 郑州轻工业学院电气信息工程学院 郑州 450002; \\ 2. 郑州轻工业学院河南省信息化电器重点实验室 郑州 450002)
}

\begin{abstract}
摘要: 针对爬壁机器人现有吸附方式的不足, 提出一种电极吸附的新方法。对电极吸附技术的吸附机理进行分析, 并根据爬 壁机器人的应用特点, 提出电极阵列式吸附结构一一共面梳状交叉电极阵列。针对该阵列结构, 基于有限元分析方法, 构建 吸附力输出模型, 并结合量子粒子群优化算法, 以电极吸附阵列吸附力输出的最大化为目标, 对电极阵列布局的参数进行优 化设计; 在对电极阵列吸附力分布特性、击穿特性等性能进行分析的基础上, 提出电极阵列结构优化设计方案, 并采用 MEMS 技术, 设计出了试验样版。为了验证电极吸附方法的有效性和所建模型的正确性, 分别基于不同的材料基底(水泥墙、玻璃 和木材), 进行吸附力测试试验, 并基于电极吸附技术, 设计单履带式攀爬机器人。试验数据及攀爬机器人的实际运行效果 表明了电极吸附技术的有效性和先进性。
\end{abstract}

关键词: 爬壁机器人; 电极吸附; 交叉电极; 有限元分析; 模型构建

中图分类号: TP24; TM7

\section{Model Construction and Optimization of Wall Climbing Robot Oriented Electro-adhesion Array}

\section{GUO Jinchao $^{1}$ CUI Guangzhao ${ }^{2}$ CHEN Xu ${ }^{1}$ GAO Yu ${ }^{1}$}

(1. School of Electrical and Information Engineering, Zhengzhou Institute of Light Industry, Zhengzhou 450002;

2. Key Lab of Information Based Appliance of Henan Province, Zhengzhou Institute of Light Industry,

Zhengzhou 450002)

\begin{abstract}
A novel electro-adhesion technology is presented for deficiency of existing adhesion methods. The mechanism of electro-adhesion is analyzed and the structure of planar combed interdigitated electrode array for climbing robots usage is presented based on the characteristics of climbing robot usage. With regard to the presented structure, the adhesion force output model is constructed with finite element analysis tool. The quantum particle swarm algorithm (QPSO) is used to optimize the parameters of the adhesion array for the maximal adhesion force output. The shape of electrode is also designed with regard to the force layout and dielectric characteristics of the whole array. With MEMS tech, a testing sample is designed and generated. In order to validate the effectiveness of electro-adhesion method, experiments were performed based on different substrates (concrete wall, glass and wood) and a single tracked climbing robot based on electro-adhesion caterpillar is designed. Experimental data and performance of the designed robot indicate effectiveness and advantage of the depicted method which can provide stable and robust adhesion foe climbing robot.
\end{abstract}

Key words: climbing robot; electro-adhesion; interdigitated electrode; finite element analysis; model construction

\section{0 前言}

要成功实现攀爬应用, 攀爬机器人必须具备三 个基本能力: 吸附、移动及载重能力, 而吸附能力

\footnotetext{
* 国家自然科学联合基金(U1304508)和河南省高校青年骨干教师支持 计划(教高[2012] 626 号)资助项目。20140512 收到初稿, 20141224 收 到修改稿
}

是机器人实现墙体攀爬的先决条件 ${ }^{[1]}$ 。当前国内外 攀爬机器人的研究主要集中在移动与吸附方式的研 究上。在攀爬机器人设计过程中, 主要采用的吸附 方式有: 负压吸附 ${ }^{[2-3]}$ 、磁力吸附 ${ }^{[4-5]}$ 、推力与夹持 力吸附 ${ }^{[6-7]}$ 及仿生吸附 ${ }^{[8-9]}$ 等。

上述的吸附方式在实际应用过程中都存在自 身的局限性，例如：真空吸附只能工作在光滑且密 封性好的壁面上, 磁性吸附只能工作在导磁材料上, 
推力与夹持力吸附方式只能工作于粗糙壁面上, 仿 生吸附方式通常制备较难且对使用环境有较高要 求。另外, 上述吸附方式中大多有一个共同的局限, 即脱附困难。真空吸附、磁力吸附、仿生吸附等吸 附方式能较快的实现吸附, 但要在运动过程中实现 较快的脱附, 则需要较多额外的能量消耗 ${ }^{[10]}$ 。

1973 年 WARDLY ${ }^{[11]}$ 首次提出静电吸盘, 因其 相对于传统吸附方式所表现出来的诸多优点, 得以 在远紫外平版印刷、液晶生产中广泛应用, 早期的 静电吸附应用大多集中在工业应用领域。2006 年, 东京工业大学的 BERENGUERES 等 ${ }^{[12]}$ 设计了一种 厘米尺寸的磁吸附仿壁虎式纤毛结构, 并首次提出 采用微型的静电吸盘所产生的电场力来代替壁虎微 观纤毛上的范德华力的概念。2007 年, 东京大学首 先提出了柔性电极的概念 ${ }^{[13]}$, 并采用柔性电路板工 艺制成了柔性电极, 通过履带方式驱动, 实现了机 器人在绝缘体壁面上的攀爬。2008 年, 美国斯坦福 大学 SRI 公司首次采用薄膜电极作为壁面移动机器 人的吸附装置 ${ }^{[14]}$, 在 $4 \mathrm{kV}$ 高压作用下, 能够在多 种材质壁面上(如金属、玻璃、粉刷墙体等)实现可 靠吸附, 吸附力最大可达 $2 \mathrm{~N} / \mathrm{cm}^{2}$ 。在国内, 哈尔 滨工业大学、北京航空航天大学等研究机构基于静 电吸附技术, 也开发出了静电吸附攀爬机器人 ${ }^{[15-16] 。 ~}$

尽管电极吸附技术拥有诸多的优点, 但对于电 极吸附阵列结构的吸附力输出模型研究较少, 对于 影响吸附力输出的各因素及相互之间的关系也没有 清晰的认识。文章将对电极吸附阵列的吸附力输出 模型构建及吸附阵列的性能优化进行探讨和研究。

\section{1 电极吸附阵列的模型构建}

常见的静电吸附装置按结构可分为: 单极型和 双极型。但鉴于攀爬机器人的可移动性和吸附性能 的稳定, 通常将静电吸附装置设计成共面梳状交叉 电极阵列的形式, 如图 1 所示。

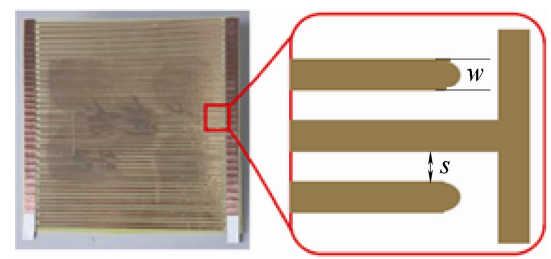

图 1 交叉梳状电极阵列结构

\section{1 电极吸附阵列的仿真分析}

为了研究电极吸附阵列的电场分布情况, 采用 Ansoft 公司的有限元分析软件 Maxwell.v13, 对电 极数分别为 $2,4,8,16$ 的梳状共面电极在真空环
境下的电场进行仿真。电极材料选择理想导体, 电 极宽度分别为: $25.000 、 12.500 、 6.250$ 和 3.125 , 电 极占空比取 0.5 , 电极间加载电压为 $1 \mathrm{kV}$, 仿真结果 如图 2 所示。从图 2 可以看出, 忽略端效应的影响, 梳状电极可以近似成电容单元的并联。根据电极阵 列在攀爬机器人应用中的特点, 为提高整体的吸附 效果, 吸附阵列上梳状电极的对数 $(N)$ 通常较大 $($ 一 般取 $N>8)$ 。这时, 端效应对吸附电极的贡献相对于

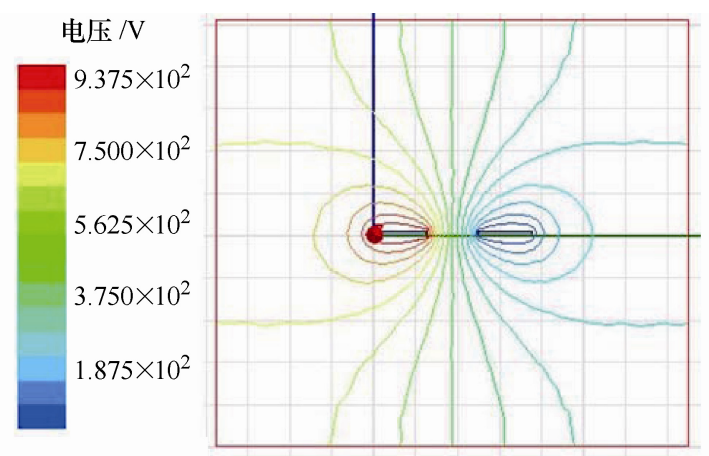

(a) 1 对共面电极

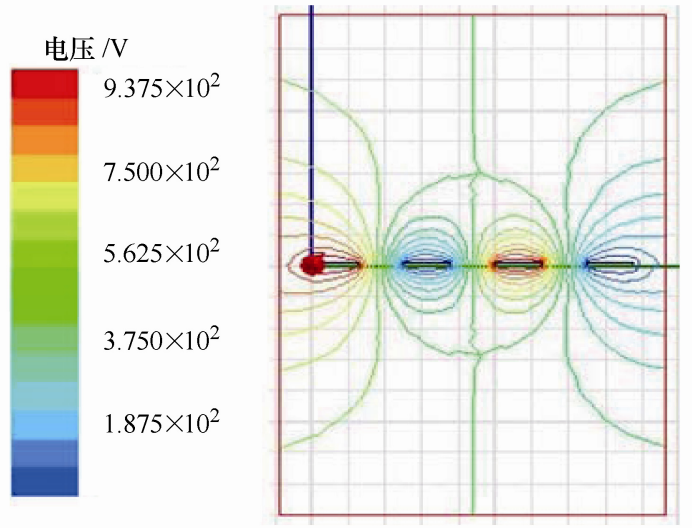

(b) 2 对共面电极

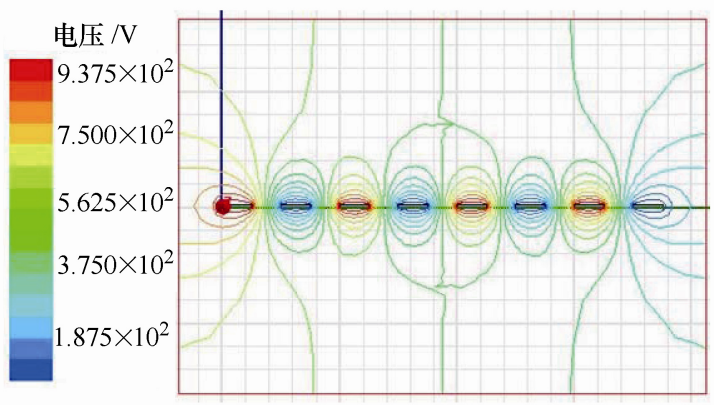

(c) 4 对共面电极

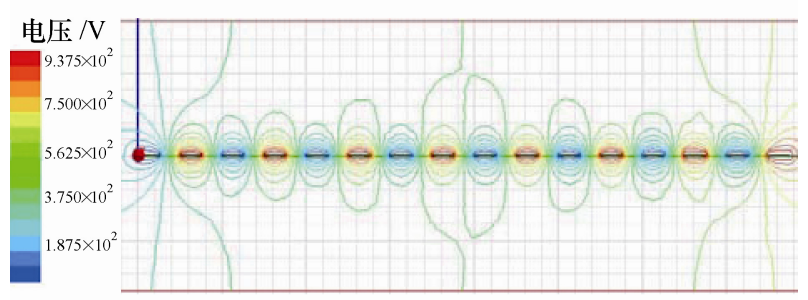

(d) 8 对共面电极

图 2 梳状共面电极有限元仿真电势场分布 
内部电极单元要小得多, 把电极阵列近似为内部电 容单元进行计算, 这样不仅可以简化计算, 同时对 阵列吸附力的计算也可控制在允许的误差范围之内。

\section{2 电极吸附阵列的仿真分析}

静电吸附包含了两种作用, 一种是库伦作用, 另一种是 J-R 效应 ${ }^{[17]}$ 。这两种作用在静电吸附过程 中同时存在, 电极与吸附壁面间的电阻率决定了哪 一种作用占主导地位。从攀爬机器人自身的安全性、 能耗及稳定性考虑, 应严格限制电极间的漏电流过 大, 因此, 在攀爬机器人应用中, 库仑作用起主导 作用。

采用 Schwarz-Christoffel 变换, 对双面共面电 极库仑作用进行分析, 单位长度电容的解析解可用 式(1)表示如下 ${ }^{[18]}$

$$
C=\frac{\varepsilon_{0} \varepsilon_{r} K\left(k^{\prime}\right)}{2 K(k)}
$$

式中, $C$ 为电容, $k 、 k^{\prime}$ 及 $K(k)$ 如式(2)、(3)、(4)所 示

$$
\begin{gathered}
k=\frac{2}{s+2 w} \\
k^{\prime}=\sqrt{1-k^{2}} \\
K(k)=\frac{\pi}{2} \sum_{n=0}^{\infty}\left[\frac{(2 n) !}{2^{2 n}(n !)^{2}}\right]^{2} k^{2 n}
\end{gathered}
$$

式中, $s$ 为电极间间距, $w$ 为电极宽度, 式(4)为第 一类椭圆积分的等价形式。

由图 2 可知, 梳状交叉电极阵列结构存在内在 的周期性, 因此, 可以用傅里叶级数来表示阵列的 电场分布, 而级数表达同时也避免了式(1)中椭圆积 分的计算过程。根据文献[19], 梳状交叉电极阵列 的势场的傅里叶级数形式为

$$
\begin{gathered}
V(x, y)=\frac{4 V_{0}}{\pi} \sum_{n=1}^{\infty} \frac{1}{2 n-1} J_{0}\left(\frac{(2 n-1) \pi s}{2 a}\right) \times \\
\sin \left(\frac{(2 n-1) \pi x}{a}\right) \exp \left(-\frac{(2 n-1) \pi|y|}{a}\right)
\end{gathered}
$$

式中, $J_{0}$ 为第一类零阶贝塞尔函数, $2 a=2(s+w)$ 为函 数周期。

根据能量守恒定律, 电极上的电场能量可由体 积分计算获得

$$
\begin{aligned}
& \frac{1}{2} C_{0}\left(2 V_{0}\right)^{2} l w=\int_{0}^{l} \int_{0}^{w} \int_{-\infty}^{\infty} \frac{1}{2} \varepsilon E^{2} \mathrm{~d} x \mathrm{~d} y \mathrm{~d} z= \\
& \int_{0}^{l} \int_{0}^{w} \int_{-\infty}^{\infty} \frac{1}{2} \varepsilon\left[\left(\frac{\mathrm{d} V}{\mathrm{~d} x}\right)^{2}+\left(\frac{\mathrm{d} V}{\mathrm{~d} y}\right)^{2}\right] \mathrm{d} x \mathrm{~d} y \mathrm{~d} z
\end{aligned}
$$

将式(5)代入式(6), 结合正余弦正交属性, 可得

$$
C_{0}=\frac{4}{\pi a} \varepsilon \sum_{n=1}^{\infty} \frac{1}{2 n-1} J_{0}^{2}\left(\frac{(2 n-1) \pi s}{a}\right)
$$

对于面积一定的电极吸附阵列, 电极对数 $N$ 为

$$
N=\frac{S}{(s+w) l}
$$

式中, $S$ 为吸附阵列的有效吸附面积, $s$ 为电极间距, $l$ 为电极长度。

因此, 交叉电极阵列的总能量

$$
\begin{aligned}
P= & \frac{1}{2} C_{0}\left(2 V_{0}\right)^{2} S= \\
& N a l \cdot \frac{8 V_{0}^{2} \varepsilon}{\pi a} \sum_{n=1}^{\infty} \frac{1}{2 n-1} J_{0}^{2}\left(\frac{(2 n-1) \pi s}{a}\right)= \\
& \frac{8 V_{0}^{2} \varepsilon l}{\pi a} N \sum_{n=1}^{\infty} \frac{1}{2 n-1} J_{0}^{2}\left(\frac{(2 n-1) \pi s}{a}\right)
\end{aligned}
$$

由式(9)可以看出, 对于面积一定的电极吸附阵 列, 其吸附功率的大小取决于电极的端电压 $V_{0}$ 、电 极对数 $(N)$ 及电极的占空比 $(s / a)$, 而这些参数从根本 上又由电极宽度 $w$ 及电极间的间距 $s$ 共同决定。

\section{2 电极吸附阵列的优化}

吸附阵列总能量的大小, 决定了输出吸附力的 大小, 该模型的建立, 为电极吸附阵列吸附力输出 的最优化设计提供了理论依据。在研究吸附阵列吸 附力输出最大化的同时, 还要充分考虑吸附阵列的 吸附力分布特性、电击穿特性、电化学稳定性及吸 附力 “吸附/脱附” 开关速度等物理、化学属性。

\section{1 电极吸附阵列的结构优化}

如前所述, 为了防止极间放电, 电极间间距存 在一个最小值 $s_{\min }$; 根据帕邢定律, 在气体恒温的 条件下, 击穿电压 $U$ 是 $p s$ 的函数 ${ }^{[20]}$

$$
U=\frac{A p s}{B+\ln (p s)}
$$

式中, $p$ 为气体压强, $s$ 为电极间离。

试验中, $p$ 取 1 个标准大气压, 当 $U$ 取 $4500 \mathrm{~V}$ 和 $3000 \mathrm{~V}$ 时, $s$ 分别为 $0.0080 \mathrm{~m}$ 和 $0.0035 \mathrm{~m}$ 。根 据式(10), 可得 $A=1.3527 \times 10^{6}, B=-4.2797$ 。

为了实现吸附阵列的最优化设计, 将电极端电 压看作是电极间距的函数

$$
V=\frac{1.3527 \times 10^{11} s}{-4.2797+5 \ln 10+\ln s}
$$

电压 $V$ 与 $s$ 的关系如图 3 所示。

由图 3 可知, 电极间距小于 $0.001 \mathrm{~m}$ 时, 外加 
电压将急速下降, 因此, 将 $s$ 与 $w$ 的值域范围分别 设为 $(0.001,0.01),(0.001,0.02)$ 。当参数 $w$ 和 $s$ 在给 定值域内变化时, 可期望获得最大的吸附功率及对 应的附加电压。

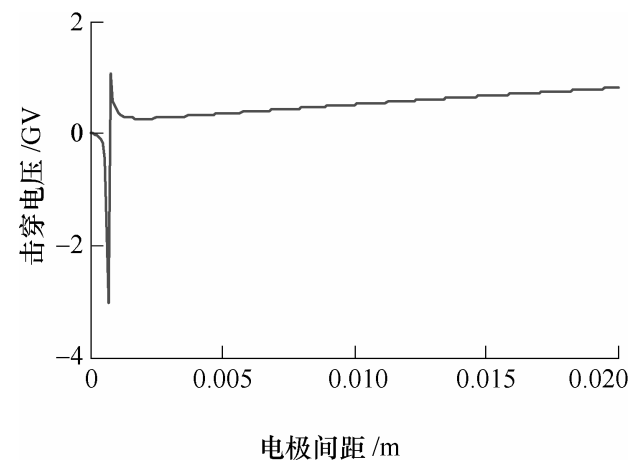

图 3 电极间击穿电压与最小间距关系图

为了实现对吸附阵列的优化设计, 采用量子粒 子群优化算法(Quantun particle swarm optimization algorithm, QPSO), 对阵列的最大输出功率进行了优 化计算, 将 $s$ 及 $w$ 作为待优化参数, 电极吸附阵列 的总能量作为代价函数, 算法参数见表 1 。

\section{表 1 因素水平表}

\begin{tabular}{lc}
\hline \multicolumn{1}{c}{ 参数 } & 数值 \\
\hline 电极阵列的有效长度 $L / \mathrm{m}$ & 0.2 \\
电极阵列的有效宽度 $W$ & 0.2 \\
粒子的认知属性值 $C_{1}$ & 0.5 \\
粒子的社会属性值 $C_{2}$ & 1.5 \\
粒子更新速度的收玫权重 $W$ & 0.9 \\
算法的迭代次数 & 30 \\
算法中采用的粒子数 & 20 \\
\hline
\end{tabular}

基于量子粒子群优化算法 ${ }^{[21]}$ 的优化过程如图 4 所示: 在第 7 代时, 即获得了最优输出, 优化后的 参数取值为 $s=w=0.001$, 最大势能 $P=6.82108$, 进 而可计算出 $N=100, V_{0}=1867.5$ 。

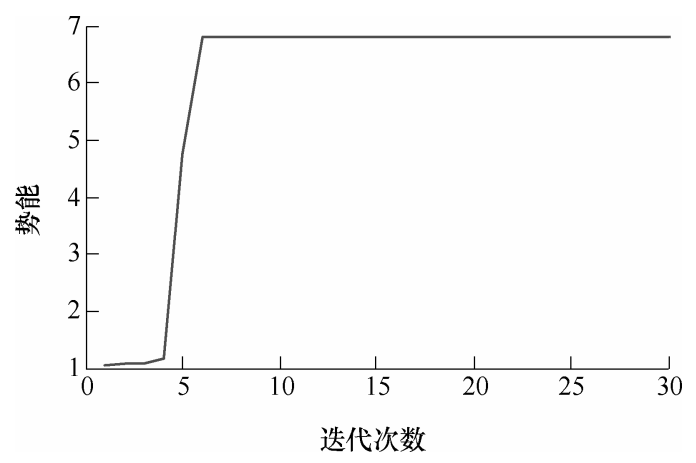

图 4 基于 QPSO 的电极阵列势能优化

\section{2 电极吸附阵列吸附力分布特性分析与优化}

由有限元仿真分析可知, 梳状电极实际上是若 干对共面双面电极的并联, 因此, 取其中的一对电 极对其电场进行分析, 进而扩展到整个梳状电极阵
列即可。利用柯西黎曼方程, 经过 Shwattes-Christiffle 逆变换, 可以得到共面双面电极 的解析解, 如式(12)所示 ${ }^{[15]}$

$$
\left\{\begin{array}{l}
|E|=\frac{U_{a}}{2 K} \frac{1}{\sqrt[4]{A^{2}+B^{2}}} \\
A=\left(x^{4}+y^{4}-6 x^{2} y^{2}\right) k^{2}-\left(1+k^{2}\right)\left(x^{2}-y^{2}\right)+1 \\
B=4 k^{2} x y\left(x^{2}-y^{2}\right)-2 x y\left(1+k^{2}\right)
\end{array}\right.
$$

式中, $U_{\mathrm{a}}$ 表示电场任一点 $a$ 处的电压, $E$ 表示 $a$ 点 的电场, $x$ 表示电极上的位置, $y$ 表示偏离电极的距 离。由于单位面积吸附力大小与电场大小平方成正 比, 因此, 对式(12)两边进行平方, 得到式(13)

$$
\left|E^{2}\right|=\left(\frac{U_{a}}{2 K}\right)^{2} \cdot \frac{1}{\sqrt{A^{2}+B^{2}}}
$$

根据式(13), 可以得到共面双面电极沿 $x$ 轴方 向的吸附力分布状况; 分别取电极占空比 $k=0.5$, 在电极厚度方向上取 $y=0.01$, 加载电压 $U_{\mathrm{a}}=1000 \mathrm{~V}$, 电场分布情况如图 5 所示。同时, 由于单位面积吸 附力正比于电场强度的平方, 图 5 亦表示了静电吸 附电极吸附力的分布状态。

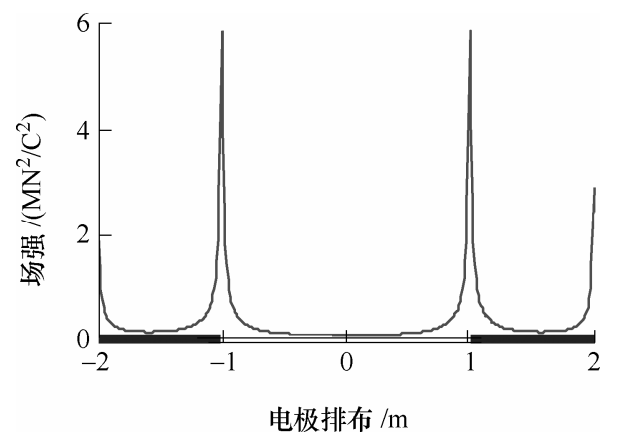

图 5 双面共面电极的吸附力分布情况

从图 5 中可以看到, 对于双面共面电极, 其区 间上的吸附力为非均匀分布，在电极的两端存在极 大值。在电极上, 吸附力先沿着电极边缘方向逐渐 减弱, 然后再在接近电极的边缘处重新出现递增, 并在电极的边缘处出现另一个吸附力的极大值。由 于梳状电极可以看成是多对双面共面电极的并联, 因此上述的吸附力分布结论可以推广到整个梳状电 极阵列。在电极上, 吸附力沿电极边缘向电极中线 递减; 而对于两电极的中间间隔位置, 吸附力从电 极端点出发逐渐向电极的间隔的中线减小。梳状电 极上的吸附力的非均匀分布, 正是由于电荷的非均 匀分布所造成的。

同时, 也可以看到，在设计上可以通过电极尺 寸的改变来调整吸附力的分布, 从而达到优化吸附 阵列的要求。一方面, 可以通过增加电极对的密度 
来使整个梳状电极上的吸附力分布更加均匀; 另一 方面, 可以根据攀爬机器人应用的特点及其动力学 性能要求 ${ }^{[22]}$, 例如足式脚掌, 通过对电极尺寸的非 均匀设计, 合理分配梳状电极的吸附力大小。

\section{3 电极吸附阵列吸附力分布特性分析与优化}

电极与壁面之间存在着一层绝缘介质, 式(12) 中 $y$ 的绝对值即代表了绝缘介质层的厚度。绝缘层 对吸附力的输出起着重要的调节作用。

取加载电压 $U_{\mathrm{a}}=1000 \mathrm{~V}$, 电极占空比值 $k=0.5$, $y$ 值分别取 $0.1 、 0.2 、 0.3$, 计算结果如图 6 所示。

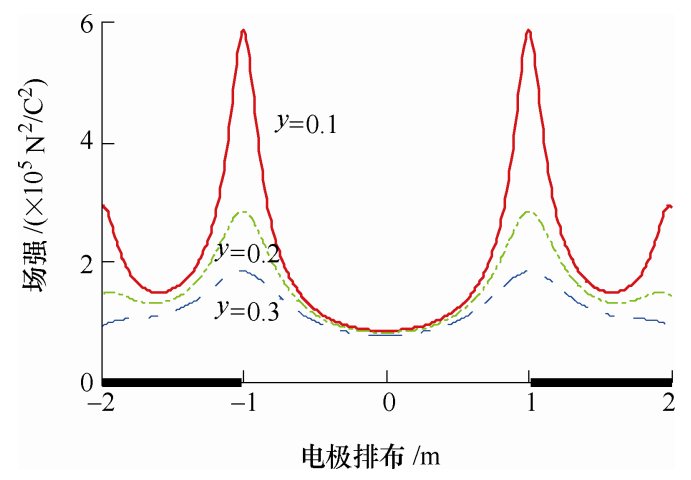

图 6 绝缘层厚度对双面共面电极吸附力的影响

从图 6 中可以看到绝缘层厚度对吸附力的影 响, 偏离 $x$ 轴距离越远, 电场值越小, 即离电极越 远, 吸附力越小。因此, 在实际设计中, 为了提高 吸附力, 可以在满足其他性能要求, 如电极绝缘层 的耐磨性的前提下, 尽可能减小绝缘介质层厚度, 也是优化吸附阵列的有效方法之一。

\section{4 电极吸附阵列边缘效应分析及优化}

当电极和电极间隔间的场强还远远低于绝缘 填充物的击穿电压时, 电极的边缘, 即从上述的电 场分析中所看到的梳状电极内单元的电极端点、外 单元的电极端点和边缘点, 却已经达到了击穿电压, 从而使绝缘填充物被击穿, 导致吸附电极的损坏, 引起吸附失效。此外, 针对吸附电极的应用特点, 还应注意壁面对电荷非均匀分布的影响, 即电极上 表面和下表面的电场的分布状况。利用有限元分析 软件对梳状电极进行仿真, 仿真结果如图 7 所示。

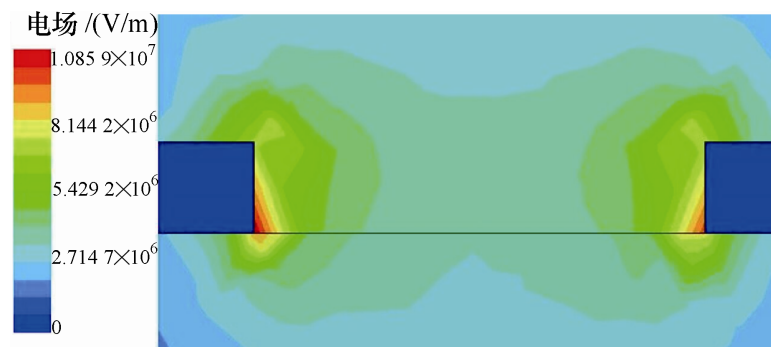

图 7 双面共面静电场有限元仿真结果

由图 7 可以看到, 在电极的端部和边缘处均存 在电荷集中现象, 场强远大于电极上的其他位置。
此外, 还可以看到, 电极边缘处由于受到壁面的影 响, 电极上下表面的场强同样分布不均。这是由于 壁面的相对介电常数远大于空气的相对介电常数, 从而电极在靠近壁面的一面电荷更加集中, 因而场 强更大。因此在实际应用中, 在梳状电极的电极端 部和边缘处需要做圆滑处理, 一方面, 可以将边缘 的直角进行圆弧过渡, 以减小电荷的集中导致电场 过大, 发生击穿。另一方面, 绝缘填充物主要需要 选择介电强度较高的材料, 尤其是电极端部和边缘 的地方。根据以上的分析, 优化后的单个电极的结 构设计示意图如图 8 所示。

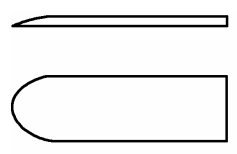

(a) 三视图

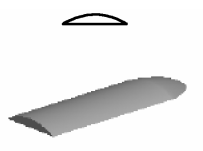

(b) 整体效果图
图 8 电极结构的优化设计

\section{3 吸附试验与分析}

为了对电极吸附阵列的吸附性能进行评估, 验 证构建模型的正确性, 项目组采用化学沉积法, 基 于最优化设计方案, 构筑了梳状交叉电极吸附阵列, 如图 1 所示。

\section{1 法向吸附力测试}

试验所用的电极吸附阵列板的大小固定为 $0.160 \mathrm{~m} \times 0.160 \mathrm{~m}$ 。为了避免电击穿的发生, 电极宽 度恒定为 $0.001 \mathrm{~m}$, 电极间距在 $(0.001 \mathrm{~m}, 0.020 \mathrm{~m})$ 之间变化; 同时, 为了观察绝缘层厚度因素对吸附 力产生的影响, 将试验阵列板根据绝缘层的厚度, 分成两个系列, 分别为 $0.10 \mathrm{~mm}$ 和 $0.35 \mathrm{~mm}$ 。同时, 为了减小吸附力试验过程中阵列板形变对吸附力产 生影响, 对阵列板基板进行了简单的硬化处理, 这 在一定程度上会降低电极阵列吸附板吸附力输出的 大小, 但却会增加试验数据的一致性, 试验中外部 施加电压固定为 $1 \mathrm{kV}$ 。基于两组吸附阵列, 分别在 不同的基底上进行测试, 每组数据采集 10 次, 去除 最大点后取平均, 法向吸附力数据如图 9 11 所示。

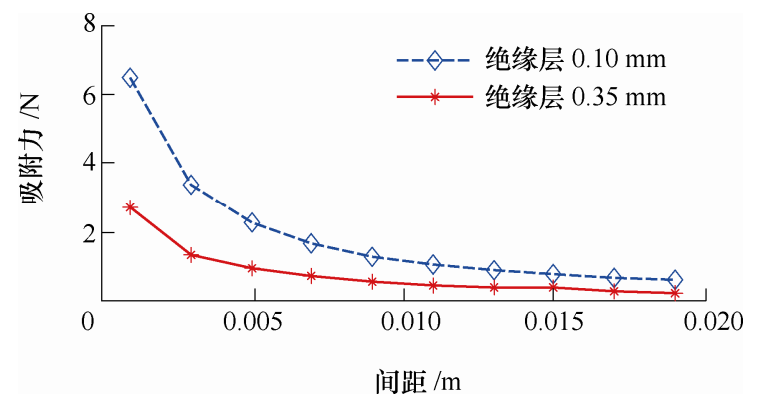

图 9 基底为水泥墙的吸附试验 


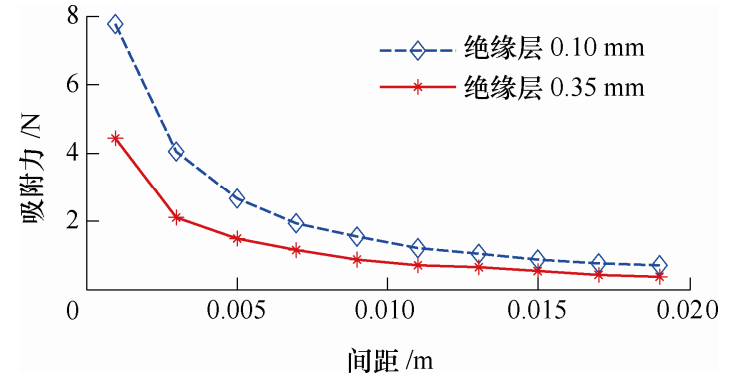

图 10 基底为玻璃的吸附试验

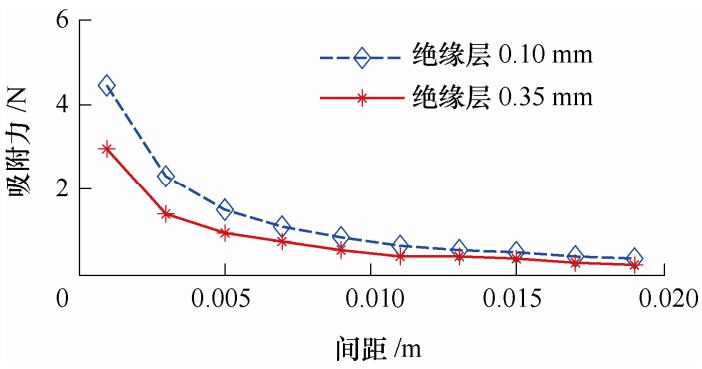

图 11 基底为木材的吸附试验

从图 9 11 中可以看出, 吸附阵列的电极间距 为 0.001 时, 法向吸附力输出最大, 这与优化的结 果相吻合; 上述组图还表明, 绝缘层对吸附力也有 着重要的影响, 吸附基底不同, 绝缘层对吸附力的 影响程度也不一样; 在水泥墙面上, 两组吸附阵列 的吸附力差别最大，玻璃上的差别居中，木材上的 差别略小于玻璃基底。这是因为, 随着绝缘层的加 厚, J-R 效应逐渐减弱, 库仑作用相对增强, 而 J-R 效应的吸附力远远大于库仑力, 同时, 随着绝缘层 的加厚, 库仑力本身也会减弱, 因此, 随着绝缘层 的加厚, 整体的吸附效果会减弱。

玻璃的介电常数大于水泥, 其上的 J-R 效应相 对较小, 对应的吸附力输出也应降低, 但试验结果 表明，在玻璃基底上的吸附力明显高于其他两种基 底, 这应该归因于玻璃良好的平滑度, 使得电极吸 附阵列能够与玻璃基底实现可靠吸附。

\section{2 基于电吸附的单履带导轨式攀爬机器人}

为了验证所获取模型的正确性, 项目组设计了 一个基于电吸附履带的导轨式攀爬机器人, 机器人 体积为: $400 \mathrm{~mm} \times 200 \mathrm{~mm} \times 30 \mathrm{~mm}$, 总质量约 $660 \mathrm{~g}$, 其移动速度不大于 $0.1 \mathrm{~m} / \mathrm{s}$, 负载不超过 $100 \mathrm{~g}$, 障 碍物高度不超过 $0.5 \mathrm{~cm}$ 。

图 12 为机器人在不同材质壁面上实现攀爬时 的抓拍组图, 验证了电极吸附技术在攀爬机器人应 用中的可行性和该方法的正确性。

\section{4 结论}

（1）针对爬壁机器人的吸附实现问题, 基于有

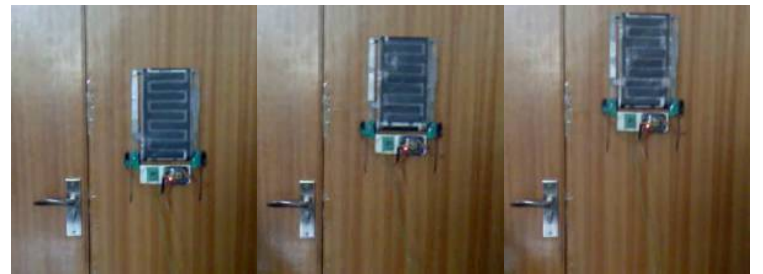

(a) 攀爬机器人在木门上

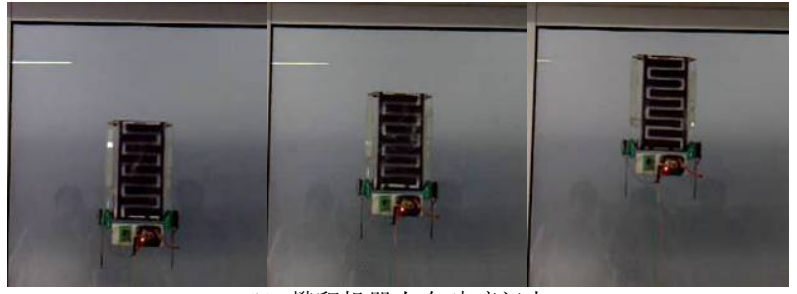

(b) 攀爬机器人在玻璃门上

图 12 攀爬机器人的攀爬试验截图

限元分析, 给出了梳状交叉电极吸附阵列结构的吸 附力模型及其近似计算方法。

（2）基于该模型，采用量子粒子群优化算法, 对电极吸附阵列结构进行了优化，并结合爬壁机器 人的自身特点, 对影响电极吸附阵列吸附性能的结 构组成、吸附能分布特性、绝缘层对吸附力的影响 等内容进行了分析, 并给出了相应的电极设计方案。

(3) 在不同的吸附基底(水泥墙面、玻璃及木材) 上进行了吸附力测试试验, 设计了基于电极吸附技 术的单轨道履带式爬壁机器人, 机器人可在多种材 质壁面上实现稳定可靠的攀爬，试验数据及攀爬机 器人的运行结果表明, 电极吸附方法具有: 吸附力 力程长、功耗低、噪声小、吸附作用范围广及自重 轻, 易于小型化设计等优点。

\section{参 考 文 献}

[1] CHU B, JUNG K, HAN CH S, et al. A survey of climbing robots: Locomotion and adhesion[J]. International Journal of Precision Engineering and Manufacturing, 2010, 11(4): 633-647.

[2] WANG Zhiheng, BAO Guanjun, ZHANG Libin, et al. Development and control of flexible pneumatic wall-climbing robot[J]. Journal of Central South University of Technology, 2009, 16(6): 961-970.

[3] LI Jun, GAO Xueshan, FAN Ningjun, et al. Adsorption performance of sliding wall-climbing robot[J]. Chinese Journal of Mechanical Engineering, 2010, 23(6): 733-741.

[4] ROCHAT F A, SCHOENEICH P A , LUTHI B A, et al. Cy-mag3D: A simple and miniature climbing robot with advance mobility in ferro-magnetic environment[J]. Industrial Robot, 2011，38: 229-233.

[5] TOSUN O, AKIN H L, TOKHI M O, et al. TRIPILLAR: Miniature magnetic caterpillar climbing robot with plane 
transition ability[C]// 12th International Conference on Climbing and Walking Robots and the Support Technologies for Mobile Machines (CLAWAR), 09-11,

September, 2009, Istanbul, Turkey. 2009: 343-350.

[6] KENNEDY B, OKON A, AGHAZARIAN H, et al. Lemur iib: A robotic system for steep terrain access[J]. Climbing and Walking Robots, 2009, 34: 1077-1084.

[7] TIN L L, YANG S X. Biologically inspired tree-climbing robot with continuum maneuvering mechanism[J]. Journal of Field Robotics， 2012(29): 843-860.

[8] MENON C, MURPHY M, SITTI M. Gecko inspired surface climbing robots[C]// International Conference on Robotics and Biomimetics, 2010, Piscataway, NJ, USA. IEEE, 2010: 431-436.

[9] YASONG L, AUSAMA A, DAN S, et al. Abigaille II: Toward the development of a spider inspired climbing robot[J]. Robotica, 2012(30): 79-89.

[10] LEI Jingtao, WANG Feng, YU Huangying, et al. Energy efficiency analysis of quadruped robot with trot gait and combined cycloid foot trajectory[J]. Chinese Journal of Mechanical Engineering, 2014, 27(1): 138-145.

[11] YAMAMOTO A, NAKASHIMA T, HIGUCHI T. Wall climbing mechanisms using electrostatic attraction generated by flexible electrodes[C]// International Symposium on Micro-Nano Mechatronics and Human Science, 2007: 389-394.

[12] BERENGUERES J, TADAKUMA K, KAMOI T, et al. Compliant distributed magnetic adhesion device for wall climbing $[\mathrm{C}] / /$ Robotics and Automation , IEEE International Conference on, 2007. IEEE, 2007 : 1256-1261.

[13] WARDLY G A. Electrostatic Wafer chuck for electron beam microfabrication[J]. Review of Scientific Instruments, 1973, 44: 1506-1509.

[14] PRAHLAD H, PELRINE R, STANFORD $\mathrm{S}$, et al. Electroadhesive robots - wall climbing robots enabled by a novel, robust, and electrically controllable adhesion technology[C]// IEEE International Conference on Robotics and Automation, 2008: 3028-3033.

[15] 黄之峰, 王鹏飞, 李满天, 等. 基于柔性静电吸附技术 的爬壁机器人研究 [J]. 机械设计与制造, 2011(6): 166-168.

HUANG Zhifeng, WANG Pengfei, LI Mantian, et al.
Study on wall-climbing robot based on flexible electrostatic attraction technique[J]. Machinery Design \& Manufacture, 2011(6): 166-168.

[16] LIU Rong, CHEN Rui, SHEN Hua, et al. Wall climbing robot using electrostatic adhesion force generated by flexible interdigital electrodes[J]. International Journal of Advanced Robotic Systems, 2013(10): 1-9.

[17] MICHAEL R S, ANDREW R M. Analysis of coulomb and Johnsen-Rahbek electrostatic chuck performance in the presence of particles for EUV lithography[C]// Proc. of SPIE, 2009: 1-14.

[18] 王黎明, 胡青春. 应用于爬壁机器人的静电吸附原理建 模及关键因素分析 $[\mathrm{J}]$. 电工电能新技术，2012(31)： 44-48.

WANG Liming, HU Qingchun. Principle modeling and key factors analysis of the electrostatic adhesion mechanism used in wall climbing $\operatorname{robot}[\mathrm{J}]$. Advanced Technology of Electrical Engineering and Energy, 2012(31): 44-48

[19] Den OTTER M W. Approximate expressions for the capacitance and electrostatic potential of interdigited electrodes[J]. Sensors and Actuators, 2002(96): 140-144.

[20] 侯清润, 茅卫红, 陈宜保. 气体放电实验与帕邢定律 $[\mathrm{J}]$. 物理实验, 2004(24): 3-4.

HOU Qingrun, MAO Weihong, CHEN Yibao. Glow discharge and Paschen law[J]. Physics Experimentation, 2004(24): 3-4.

[21] YU Ying, LI Yongsheng, YU Xiaochun. Application of particle swarm optimization in the engineering optimization design[J]. Chinese Journal of Mechanical Engineering, 2008, 44(12): 226-231.

[22] 熊有伦, 丁汉. 机器人动力学性能指标及其优化 $[\mathrm{J}]$. 机 械工程学报, 1989, 25(2): 9-15.

XIONG Youlun, DING Han. Performance index and optimization of dynamics in manipulator arm[J]. Chinese Journal of Mechanical Engineering, 1989， 25(2): 9-15.

作者简介: 过金超, 男, 1978 年出生, 博士, 副教授, 硕士研究生导师。 主要研究方向为多机器人系统及智能机器人的开发与设计。

E-mail: gjc@zzuli.edu.cn

崔光照, 男, 1957 年出生, 博士, 教授。主要研究方向为无线传感器网 络、生物计算及工业机器人。

E-mail: cgzh2008@163.com 